LA-UR-01-3050

Approved for public release; distribution is unlimited.

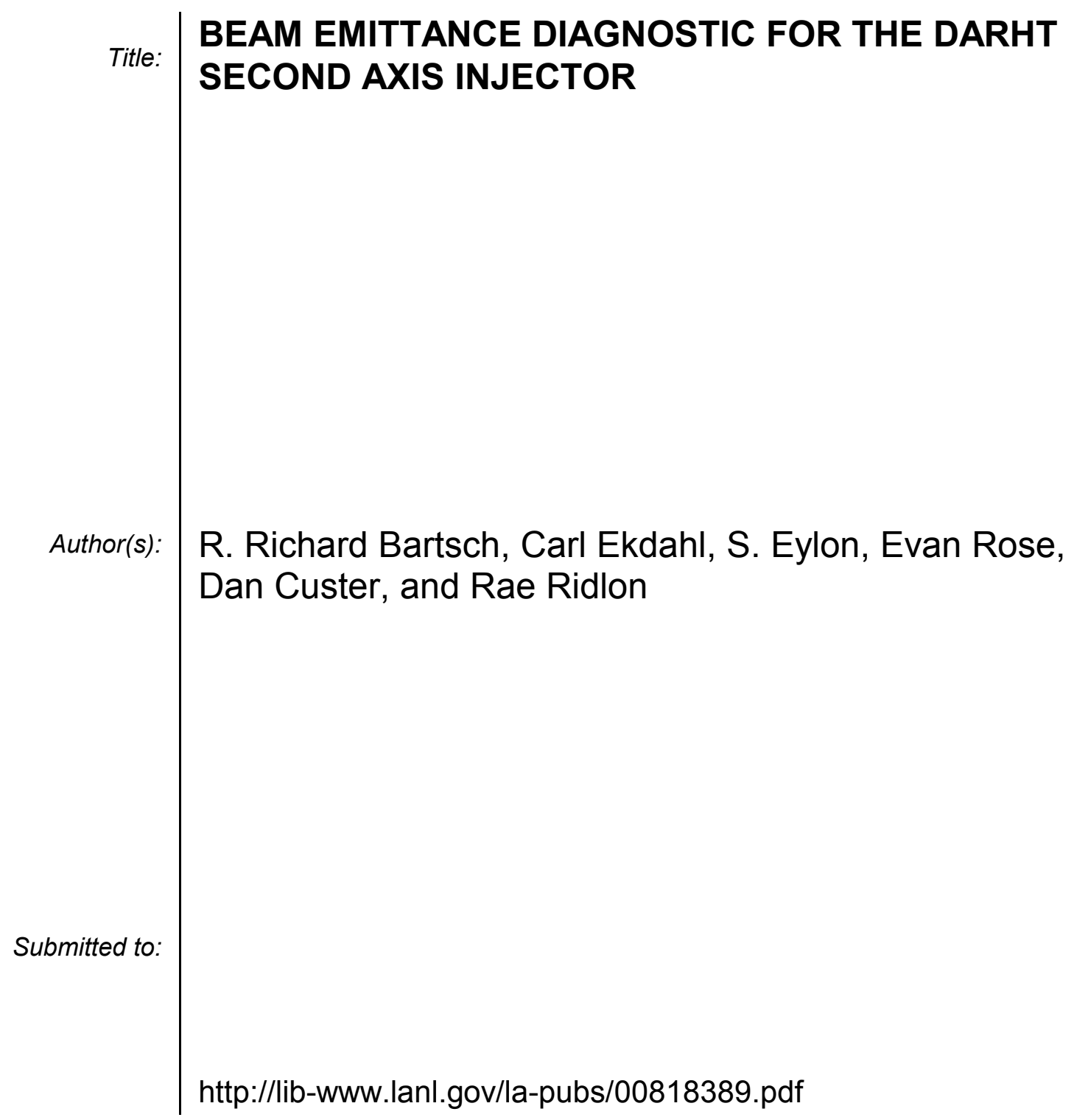

Los Alamos National Laboratory, an affirmative action/equal opportunity employer, is operated by the University of California for the U.S. Department of Energy under contract W-7405-ENG-36. By acceptance of this article, the publisher recognizes that the U.S. Government retains a nonexclusive, royaltyfree license to publish or reproduce the published form of this contribution, or to allow others to do so, for U.S. Government purposes. Los Alamos National Laboratory requests that the publisher identify this article as work performed under the auspices of the U.S. Department of Energy. Los Alamos National Laboratory strongly supports academic freedom and a researcher's right to publish; as an institution, however, the Laboratory does not endorse the viewpoint of a publication or guarantee its technical correctness. 


\title{
BEAM EMITTANCE DIAGNOSTIC FOR THE DARHT SECOND AXIS INJECTOR
}

\author{
R. Richard Bartsch, Carl Ekdahl, S. Eylon,* \\ Evan Rose, Dan Custer, and Rae Ridlon \\ LANL, Los Alamos, N.M. 87544, *LBL, Berkeley, CA 95720
}

\section{Abstract}

Low beam emittance is key to achieving the required spot size at the output focus of the DARHT Second Axis. The nominal electron beam parameters at the output of the injector are $2 \mathrm{kA}, 4.6 \mathrm{MeV}, 2$-microsecond pulse width and an rms radius less than $1 \mathrm{~cm}$. Emittance is measured by bringing the beam to a focus in which the emittance is a dominant influence in determining the spot size. The spot size is measured from Cerenkov or optical transition radiation (OTR) generated from a target intercepted by the beam. The current density in the focused DARHT beam would melt this target in less than $1 / 2$ microsec. To prevent this we have designed a DC magnetic transport system that defocuses the beam on the emittance target to prevent overheating, and uses a 125-ns half period pulsed solenoid to selectively focus the beam for short times during the beam pulse.

During the development of the fast-focusing portion of this diagnostic it has been determined that the focusing pulse must rapidly sweep through the focus at the target to an over-focused condition to avoid target damage due to overheating.

The fast focus produces $\sim 1$ kilogauss field over an effective length of $\sim 50 \mathrm{~cm}$ to bring the beam to a focus on the target. The fast focus field is generated with a 12turn coil located inside the beam-transport vacuum chamber with the entire fast coil structure within the bore of a D.C. magnet. The pulsed coil diameter of $\sim 15 \mathrm{~cm}$ is dictated by the return current path at the nominal vacuum wall. Since the drive system is to use $40 \mathrm{kV}$ to $50 \mathrm{kV}$ technology and much of the inductance is in the drive and feed circuit, the coil design has three 120 degree segments.

The coil, driver and feed system design, as well as beam envelope calculations and target heating calculations are presented below. Operation of the OTR imaging system will be discussed in separate publication (Ref. 1).

\section{BEAM PROFILE AND EMITTANCE MEASUREMENTS ON DARHT-II}

During commissioning experiments on the DARHT-II accelerator we must measure the electron beam radial current density profile and the beam emittance. The usual method for measuring beam current profiles is to image beam-produced radiation from a target inserted into the beam. This radiation can be visible Cerenkov radiation, optical transition radiation (OTR), or bremsstrahlung radiation. The beam emittance can be derived from a series of such measurements at a single location if the magnetic focusing of the beam is varied. The envelope equation for a beam focused by a solenoidal-magnet thin lens can be solved analytically to find the beam radius at the imaging location as a function the emittance, current, energy, initial radius at the lens, initial convergence angle, and the lens focal length (which is inversely proportional to the square of the magnetic field at the lens center). In general, all of the parameters in this set are known except for the emittance, initial radius, and convergence angle. In principle, three measurements of radius with different magnet settings (focal lengths) are sufficient to determine these unknowns. In practice, the solenoidal transport is far from the thin lens idealization, so several magnet settings are typically used to generate a table of radius vs. magnet setting. A least squares fit of transport equation solutions to these data yields the emittance, as well as the two initial conditions. This procedure yields the most accurate results if the set of measured radii includes the beam waist, because the ratio of the emittance term in the envelope equation to the space-charge term scales inversely with the square of the radius, so the radius is most sensitive to emittance when it is small.

This presents a real challenge for DARHT-II because of the long beam pulse. The DARHT-II accelerator will produce a $2-\mathrm{kA}, 2-\mu \mathrm{s}$ beam pulse, and can be expected to have a beam waist radius less than $1 \mathrm{~cm}$ at the imaging location. For example, the energy deposited by a 2-kA, 1$\mathrm{cm}$ radius beam is sufficient to melt steel in less than onehalf microsecond, and would certainly destroy any imaging target inserted in its path. Moreover, it has been shown experimentally that a target surface-temperature rise of only 200 degrees $\mathrm{C}$ is enough to produce spacecharge neutralizing ions that lead to disruption of the beam, thereby invalidating the measurement. To prevent this, we will use the fast-focus solenoid described below to rapidly pinch the beam down from a defocused radius large enough that the target is not overheated. To prevent overheating, the pinch and subsequent relaxation must occur in about 100 ns. In Fig. 1(a) and (b) we show the 4.6 MeV DARHT-II injector beam radius and heating that would result from our baseline design fast-focus coil pulsed to a peak field of 600 Gauss with a half cycle time of 100-ns. For this calculation the beamline magnets were tuned using our XTR envelope code to produce a 
beam $\sim 5-\mathrm{cm}$ radius without energizing the fast coil. The greatest heating will occur when we pulse the fast-focus coil at the end of the $2-\mu \mathrm{sec}$ current pulse, as was assumed for this calculation. It is seen from the plot that the temperature rise during the fast pinch is well below the 200-degree threshold for beam disruption.

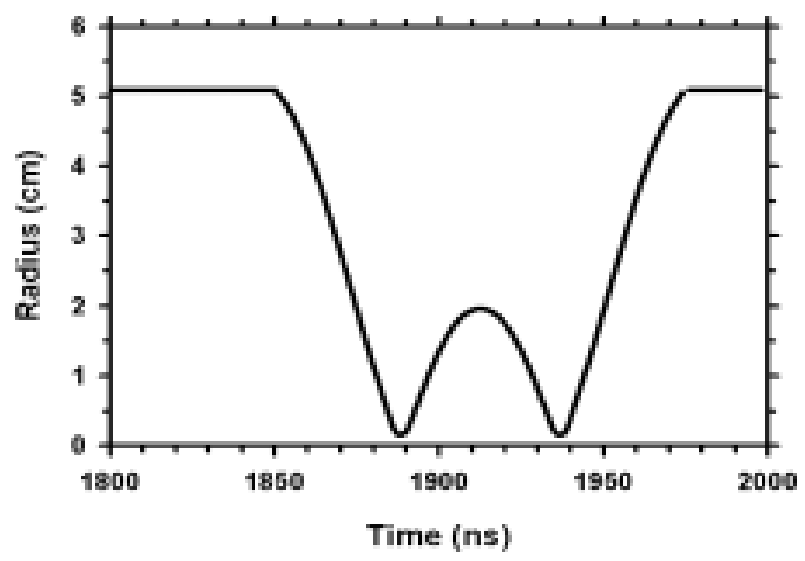

Figure 1(a): DARHT Second Axis Beam Radius vs. Time at the OTR Target During Pulsed Focussing

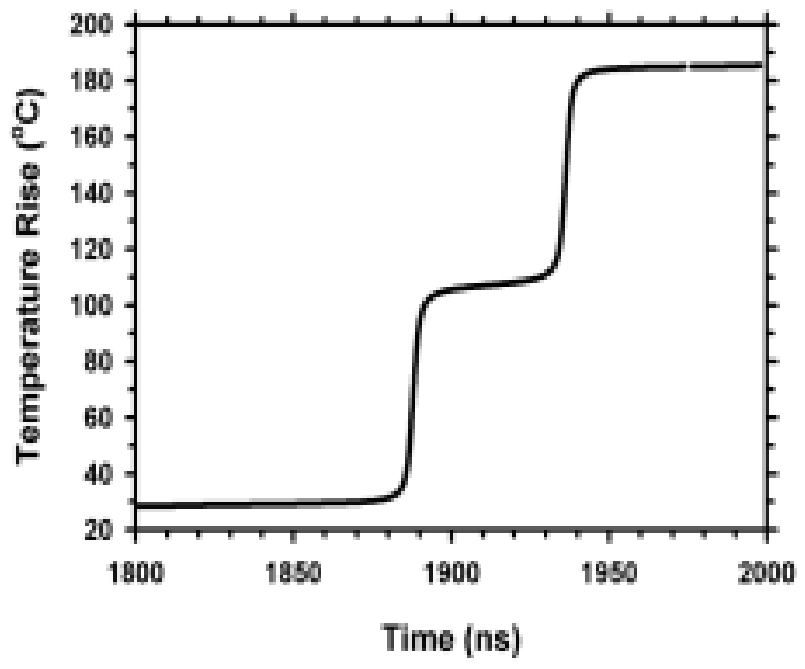

Figure1(b): OTR Target Heating During Pulsed Focusing

An obvious advantage of the fast-pinch is that it generates a huge database of radii vs magnetic field on a single shot compared with the shot-to-shot method of changing a magnet setting. This should result in much improved accuracy for the emittance measurements.

\section{FAST-FOCUS MAGNET DESIGN}

The pulsed magnet is required to provide a peak field of up to 1 kilogauss over an axial extent of 0.5 meter to achieve the focusing performance dictated by target heating described in Part I. Since the driver is a switched array of capacitors with low damping, the designing halfcycle time is 125 nanosec. The use of 40 to $50 \mathrm{kV}$ (max) driver technology with achievable external circuit inductance has resulted in a coil array of 1/3-turn segments fed in parallel. A single turn is shown in Fig. 2. A cutaway side view of the entire magnet assembly is shown in Fig. 3. A dc-magnet is located outside the vacuum wall of the pulsed-magnet assembly and is the ultimate mechanical constraint on the outer diameter of the fast-focus coil return flux region. The pulsed magnet coils are $\sim 15 \mathrm{~cm}$ i.d. and are fed axially with vacuum strip lines near the outer wall. Vacuum feed through headers are located on either side of the d.c. magnet to permit connection of up to 30 coaxial cables to drive each half of the pulsed magnet. Individual turns are "clocked" to permit the internal, axial feeds to nest. Not shown in the cutaway is an axially slotted cylinder extending through the pulsed-coil at the nominal diameter of the DARHT $2^{\text {nd }}$ axis vacuum wall. This axial array of bars carries the return current of the beam and permits the penetration of axial flux to the position of the beam. It also selects against some of the unwanted fields associated with the pulsed magnet feeds.

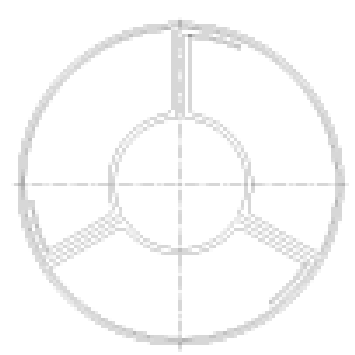

Figure 2: Segmented Pulsed-Focusing Coil

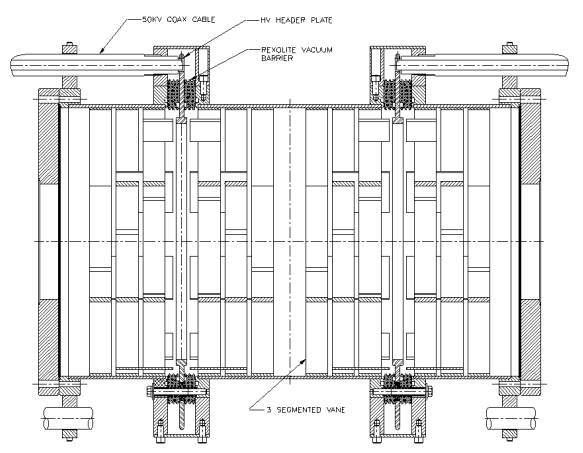

Figure 3: Side View of 12-turn Pulsed-Focusing Coil Array 
The computer code FLUX2D (Magsoft Corp. Troy, New York) has been utilized to optimize the coil design and determine the required drive conditions. For the purpose of surveying a wide variety of cases an equi-flux solution (assuming equal voltage on all coils) has been utilized. It is possible to couple a driven-circuit to numerically solve for the current distribution, however in practice it has proven easier to iterate on the flux in each coil until approximately self-consistent currents and voltages appear on each turn allowing for the differing feed inductances from the header to each coil. For the final design the axial field and flux contours are shown in Fig. 4 (a) and (b). The line integral of magnetic field around each conductor gives the peak current and the specified flux gives the peak loop voltage.

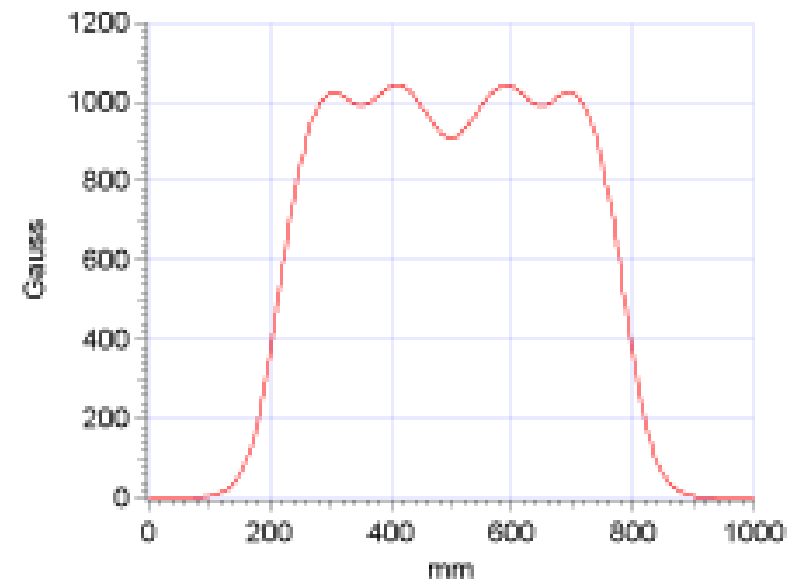

Figure 4(a): Calculated Axial Magnetic Field Profile for Maximum Drive Current (100kA)

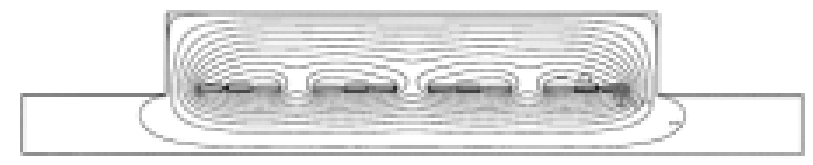

Figure 4(b): Calculated Constant Flux Contours-half view, bottom border is the pulsed-magnet axis

At each of the two headers the total voltage, feed inductance times di/dt plus $\mathrm{d}$ (flux)/dt, is $\sim 21 \mathrm{kV}$ and the net current is 100kA. The 30 parallel cables plus header on each half of the coil have an estimated inductance of $5 \mathrm{nH}$. Since the radian frequency is $25 \times 10^{6} / \mathrm{sec}$, the equivalent inductance at each driver in the array of drivers on each half of the coil is 13.4 nanohenries times N, where $\mathrm{N}$ is the number of drivers per half of the pulsed coil. $\mathrm{N}$ is conveniently 6 or 10 , but can be 8 with a change in header.

\section{PULSED-POWER DRIVER DESIGN}

Minimization of inductance is a key issue in utilizing available 40 to $50 \mathrm{kV}$ components in a pulsed-power driver system that matches the fast-focus magnet described above. Several prototype drivers, consisting of an array of $2.7 \mathrm{nF}$ Murata ceramic capacitors, a fast switch and an array of shorted h.v. coaxial load cables, have been evaluated in low inductance mounting configurations. A TRITON model F228 thyratron has been evaluated with series h.v. diodes to end the L-C discharged at the half-cycle time (Ref 2). A $100 \mathrm{kV}$ TITAN-PSI (Maxwell) and a $50 \mathrm{kV}$ TITAN-PSI (Maxwell) spark gap have each been evaluated without circuitry to end the ringing discharge at the half-cycle time. Primarily because of the low inductance of the short, $50 \mathrm{kV}$ gap it has been chosen as the fast switch for this system (TITAN-PSI Maxwell) Part No. 40044). The test configuration for this gap is shown in Fig. 5. Current was observed with a current monitor transformer on one of the load cables and with a B-dot probe in an annular groove in the spark gap mount. The length of the three RG-220 load cables was trimmed to obtain half-cycle times of 120-125 nanosec. Tests were conducted up to the $40 \mathrm{kV}$ nominal maximum rating of the ceramic capacitors (5 in parallel) and peak currents up to $12.7 \mathrm{kA}$ were achieved. The inductance of the $50 \mathrm{kV}$ spark gap, capacitors and mount was determined to be $\sim 40 \mathrm{nH}$. Some minor variation in the half period with charge voltage was observed which was opposite to the expected trend from the voltage variation of the ceramic capacitors. This is shown in Fig. 6.

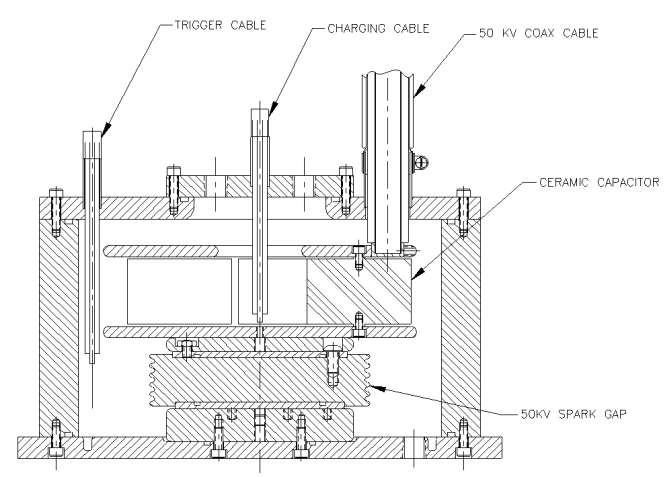

Figure 5: Spark Gap, Ceramic Capacitor (5ea) Test Unit 


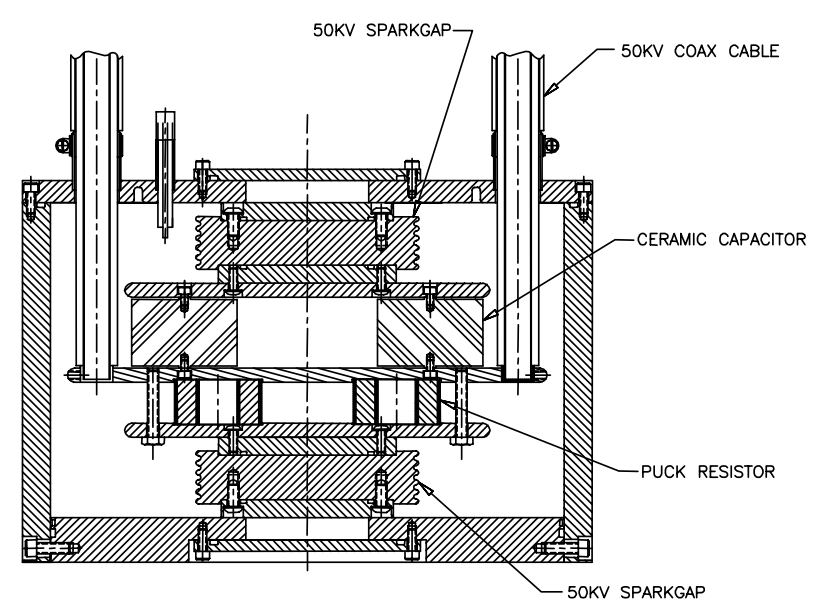

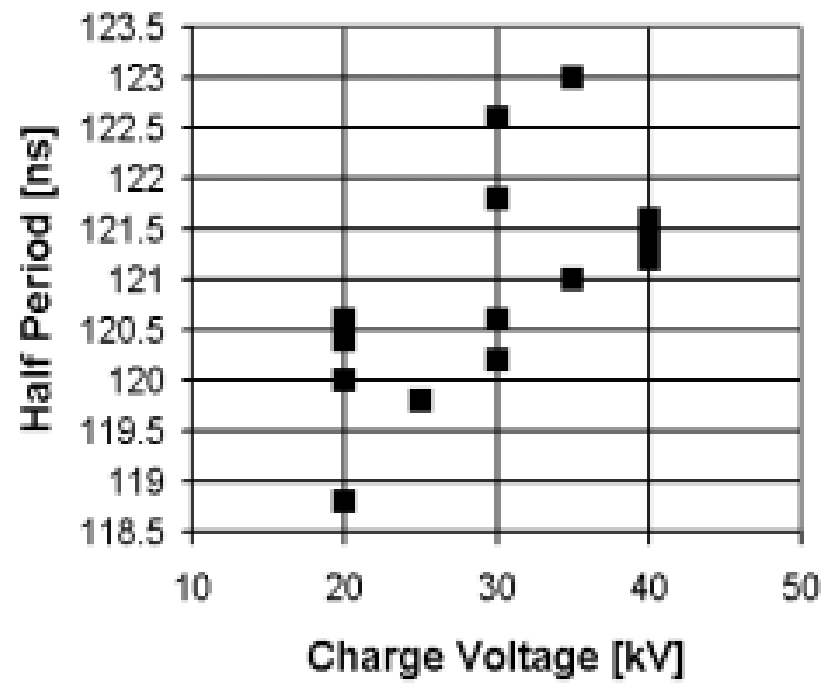

Figure 6: Half-Period vs. Charge Voltage for Spark Gap Test Unit

\section{FAST-FOCUSING SYSTEM}

The base-line fast-focus system consists of the dualheader coil of Section II above with 304.5 foot-long cables on each end (Dielectric Sciences 2250-50kV, $26 \mathrm{nH} / \mathrm{ft}, 17 \mathrm{ohm})$ and 10 high-voltage drivers on each end. Each driver will be configured with a second spark gap to enable resistive damping of the back-charged capacitor array at the half-cycle time. This driver configuration is shown in Fig. 7. Each driver unit can accommodate up to 6 capacitors (TDK model FHV$12 \mathrm{AN}, 2.1 \mathrm{nF}, 50 \mathrm{kV}$ ), although present operations can be accommodated with 4 or 5 capacitors per driver. The half period is 120 nanosec for 4 capacitors and 134 nanosec for 5 capacitors. The option to use diodes to passively cutoff the waveform at the half-period has been retained. Hardware is presently being fabricated for installation on the DARHT $2^{\text {nd }}$ axis.
Figure 7: Dual-Spark-Gap, Fast-Coil Driver Unit

\section{REFERENCES}

[1] Ken Moy, to be published.

[2] S. Eylon, et. al., "Time dependant beam focusing of the DARHT II injector diode," Proc. 1999 Particle Accelerator Conference, New York, NY, 1999.

This work was performed on US DOE Contracts W-7405ENG-48 and W-7405-ENG-36. 